\title{
Klassisch atomistische Energieberechnung null- und eindimensionaler Gitterstörungen
}

\begin{abstract}
Von F. WAHL
Aus dem Institut für theoretische und angewandte Physik der Technischen Hochschule Stuttgart

(Z. Naturforschg. 15 a, 616-625 [1960] ; eingegangen am 23. März 1960)

Zur Berechnung der Verzerrungsenergie von Störstellen, die man mit Hilfe der klassischen nichtlinearen Gitterstatik behandelt, wird eine Methode angegeben. Sie besteht in der Ersetzung des quadratischen Verzerrungsanteils im Energieausdruck durch die höheren Kraftglieder, was sich zufolge des Kräftegleichgewichts zwischen linearer und nichtlinearer Gitterreaktionen durchführen läßt. Da die nichtlinearen Gitterreaktionsanteile räumlich auf wenige, ausgezeichnete Bereiche beschränkt sind, wird das Problem der Summation der Teilchenpotentiale über das gesamte Verschiebungsfeld reduziert auf eine Summation, welche nur die Freiheitsgrade jener Stellen enthält, an denen die Nichtlinearitäten für den Gleichgewichtszustand wesentlich werden. Bei nulldimensionalen Störstellen sind diese auf die Umgebung der Störstellen allein konzentriert. Die Betrachtungen verlaufen hier sehr einfach. Bei eindimensionalen Störstellen spielen die Vorverschiebungen eine wesentliche Rolle, so daß die Nichtlinearitäten zunächst nicht ohne weiteres am Versetzungskern allein angenommen werden dürfen. In diesem Fall lassen sich die Betrachtungen nur am speziellen Modell anstellen Als Modelle werden Schrauben- und Stufenversetzungen untersucht.
\end{abstract}

In den vorhergehenden Arbeiten ${ }^{1}$, die sich mit der klassischen nichtlinearen Gitterstatik beschäftigen, und die deren Anwendbarkeit auf null- und eindimensionale Störkonfiguration vom theoretischen Standpunkt aus untersuchen, fehlt noch eine zusammenhängende Methode der Energiebestimmung. Selbstverständlich ist es vom prinzipiellen Standpunkt aus sehr einfach, die Energie eines verzerrten Kristalls anzugeben. Da die Auslenkungen der Gitterbausteine in einer gestörten Kristallstruktur aus den gitterstatischen Rechnungen bekannt sind, müssen nur die Wechselwirkungspotentiale für die Relativabstände angeschrieben werden. Im allgemeinen wird sich aber ein elastisches, oder auch plastisches Verschiebungsfeld sehr weit ausdehnen. Die Aufsummation aller Wechselwirkungspotentiale ist dann nicht mehr ohne weiteres durchführbar. Man wird daher versuchen, die Summe durch Einführung der aus den gitterstatischen Rechnungen bekannten Kräfte, oder der durch Vorverschiebungen erzeugten „Zwangskräfte“, auf handliche Ausdrücke zu reduzieren. Dies gelingt auf Grund des Kräftegleichgewichts zwischen der linearen Gitterreaktion einerseits, den äußeren Kräften und der nichtlinearen Gitterreaktion andererseits. Solange die nichtlinearen Anteile auf kleine Kristallbereiche lokalisiert sind, lassen sich die Überlegungen schnell erledigen; der Problemkreis der nulldimensionalen Störungen ist

1 E. Fues u. H. Stumpf, Z. Naturforschg. 10 a, 1055 [1955]. E. Fues, H. StumpF u. F. W [1958]. - H. Gross u. F. WAHL, Z. Naturforschg. 14 a, 285

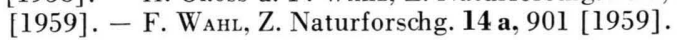

damit vollständig erfaßt. Anders ist dies bei eindimensionalen Störkonfigurationen. Insbesondere die Bestimmung der Verzerrungsenergie von Versetzungen bedarf einer tieferen Untersuchung, da bei ihnen elastische und plastische Deformationen gleichermaßen beteiligt sind. Der weitaus größere Teil der vorliegenden Arbeit wird sich mit solchen weitausgedehnten Störungen beschäftigen. Dies ist oft vom allgemeinsten Standpunkt aus nicht mehr möglich, so daß wir nicht umhin können, spezielle Modelle heranzuziehen. Der Energieberechnung von Schrauben- und Stufenversetzung soll daher in besonderem Maße unsere Aufmerksamkeit gelten.

\section{$\S 1$. Verzerrungsenergie bei nulldimensionalen Störkonfigurationen}

Solange die Berechnung von Störungen des idealen Kristallaufbaus nur in kleinen, scharf lokalisierbaren Bereichen des Kristalls eine Berücksichtigung nichtlinearer Kraftglieder fordert, verlaufen die Betrachtungen zur Bestimmung der Verzerrungsenergie verhältnismäßig einfach. Wir können sie in diesem Paragraphen erledigen.

Dazu gehen wir aus von der potentiellen Energie eines Kristalls. Sie ist eine Funktion der Relativabstände $\left|\Re_{m}-\Re_{i}\right|$ aller Gitterbausteine ${ }^{2}$

$$
U=\frac{1}{2} \sum_{i \neq m} \sum_{i m}\left(\left|\Re_{m}-\Re_{i}\right|\right)
$$

Diese Arbeiten sollen der Reihe nach mit (I) bis (IV) bezeichnet werden.

2 Von Nichtzentralkräften wird abgesehen. Wir schreiben der Einfachheit halber Vektorindizes (siehe auch III). 
- Entwickeln wir die Wechselwirkungspotentiale $P_{\text {im }}$ in (1) nach den Auslenkungen aus den idealen Gitterlagen $\vec{\xi}_{\mathfrak{m}}=\Re_{\mathfrak{m}}-\Re_{\mathfrak{m}}^{0}$, so erhalten wir als Ausdruck für die Verzerrungsenergie

$$
\begin{array}{r}
U=\frac{1}{2} \sum_{\mathrm{i} \neq \mathrm{m}} \sum_{\mathrm{m}}\left\{P_{\mathrm{im}}^{0}+\frac{1}{2}\left[\overrightarrow{\xi_{\mathrm{i}}}-\overrightarrow{\xi_{\mathrm{m}}}\right] \cdot \boldsymbol{A}_{\mathrm{im}} \cdot\left[\vec{\xi}_{\mathrm{m}}-\vec{\xi}_{\mathrm{i}}\right]\right. \\
\left.+P_{\mathrm{im}}^{\mathrm{h}}\left(\vec{\xi}_{\mathrm{m}}-\vec{\xi}_{\mathrm{i}}\right)\right\} .
\end{array}
$$

$P_{\mathrm{im}}^{\mathrm{h}}\left(\vec{\xi}_{\mathrm{m}}-\vec{\xi}_{\mathfrak{i}}\right)$ ist dabei die Gesamtheit der höheren Glieder, die wir nicht weiter angeben. Die linearen Glieder in (2) verschwinden aus Stabilitätsgründen.

Bei Einwirkung äußerer Kräfte ${ }^{3} \mathfrak{f}_{i}\left(\vec{\xi}_{\mathfrak{t}}\right)$ wird dann die Lage der Gitterbausteine gegeben durch Gleichgewichtsbedingungen, die wir aus (2) durch Differentiation erhalten

$$
\begin{aligned}
\mathfrak{f}_{\mathfrak{i}}\left(\boldsymbol{\xi}_{\mathfrak{t}}\right)= & \nabla_{\mathfrak{i}} U \\
& =\sum_{\mathfrak{m}}\left\{\boldsymbol{A}_{\mathrm{im}} \cdot\left[\vec{\xi}_{\mathrm{m}}-\vec{\xi}_{\mathfrak{i}}\right]+\frac{1}{2} \nabla_{\mathfrak{i}} P_{\mathfrak{i m}}^{\mathrm{h}}\left(\vec{\xi}_{\mathfrak{m}}-\vec{\xi}_{\mathfrak{i}}\right)\right\} \\
& =\sum_{\mathfrak{m}}\left\{\boldsymbol{A}_{\mathfrak{i m}} \cdot\left[\vec{\xi}_{\mathfrak{m}}-\vec{\xi}_{\mathfrak{i}}\right]+\mathfrak{f}_{\mathfrak{i m}}^{\mathrm{h}}\left(\xi_{\mathfrak{m}}-\vec{\xi}_{\mathfrak{i}}\right)\right\} .
\end{aligned}
$$

Zur Berechnung der neuen Ruhelagen gehen wir dann stets von der Form

$$
\sum_{\mathrm{m}} \boldsymbol{A}_{\mathrm{im}} \cdot\left[\vec{\xi}_{\mathrm{m}}-\vec{\xi}_{\mathrm{i}}\right]=\left\{\mathfrak{f}_{\mathrm{i}} \cdot\left(\vec{\xi}_{\mathfrak{t}}\right)-\sum_{\mathrm{m}} \mathfrak{f}_{\mathrm{im}}^{\mathrm{h}}\left(\vec{\xi}_{\mathrm{m}}-\vec{\xi}_{\mathrm{i}}\right)\right\}
$$

aus. Führen wir die Umkehrung durch, so finden wir für die Auslenkungen eine Gleichung

$$
\vec{\xi}_{\mathfrak{m}}=\sum_{i} B_{\mathfrak{m i}} \cdot\left[\mathfrak{f}_{\mathfrak{i}}\left(\vec{\xi}_{\mathfrak{t}}\right)-\sum_{\mathfrak{m}} \mathfrak{f}_{\mathfrak{i m \mathfrak { l }}}^{\mathrm{h}}\left(\vec{\xi}_{\mathfrak{m}}-\vec{\xi}_{\mathfrak{i}}\right)\right],
$$

die im allgemeinen nur durch Iteration gelöst werden kann, da nicht nur die $\mathfrak{f}_{\mathrm{im}}^{\mathrm{h}}$, sondern häufig auch die äußeren Kräfte $\mathfrak{f}_{\mathfrak{i}}\left(\xi_{\mathfrak{f}}\right)$ selbst noch von den Auslenkungen abhängen.

Will man nun die Energie berechnen, die als Verzerrungsenergie durch die Verschiebung von Kristallbausteinen gegenüber dem Idealzustand zusätzlich aufgenommen wird, so ist es sehr zweckmäßig, den Energieausdruck (1) mit Hilfe der Kraftgleichungen (4) umzuformen. Dazu müssen wir bedenken, daß nur im Gleichgewichtsfall die Gln. (4) durch eindeutig bestimmte Verschiebungen erfüllt sind, und da $\beta$ eben diese Verschiebungen in den Energieaus-

\footnotetext{
3 Als äußere Kräfte wollen wir alle diejenigen Kräfte verstehen, die durch Wechselwirkung mit Systemen entstehen, die ursprünglich nicht im betrachteten Kristall enthalten sind.

4 Das nächstliegende wäre, die Kräfte gleich mit den Differenzen $\xi_{i}-\xi_{m}$ zu multiplizieren. Auf der linken Seite von
}

druck (1) eingesetzt werden müssen. Da in diesem Fall (1) und (4) simultan gelten, können wir das quadratische Glied auf der rechten Seite von (1) ersetzen durch einen Ausdruck, den wir erhalten, wenn wir die rechte Seite von (4) mit $\frac{1}{2} \vec{\xi}_{i}$ multiplizieren und über $\dot{i}$ summieren ${ }^{4}$

$$
\begin{aligned}
& \sum_{i} \frac{1}{2} \vec{\xi}_{\mathrm{i}} \cdot\left[\mathfrak{f}_{\mathrm{i}}\left(\vec{\xi}_{\mathfrak{t}}\right)-\sum_{\mathrm{m}} \mathfrak{f}_{\mathrm{im}}^{\mathrm{h}}\left(\vec{\xi}_{\mathrm{m}}-\vec{\xi}_{\mathrm{i}}\right)\right] \\
& \quad=\frac{1}{2} \sum_{i, m}^{\prime} \vec{\xi}_{\mathrm{i}} \cdot \boldsymbol{A}_{\mathrm{im}} \cdot\left[\vec{\xi}_{\mathrm{m}}-\vec{\xi}_{\mathrm{i}}\right] \\
& =\frac{1}{4} \sum_{\mathrm{i}, \mathrm{m}}^{\prime}\left\{\vec{\xi}_{\mathrm{i}} \cdot \boldsymbol{A}_{\mathrm{im}} \cdot\left[\vec{\xi}_{\mathrm{m}}-\vec{\xi}_{\mathrm{i}}\right]+\vec{\xi}_{\mathrm{m}} \cdot \boldsymbol{A}_{\mathrm{mi}} \cdot\left[\vec{\xi}_{\mathrm{i}}-\vec{\xi}_{\mathrm{m}}\right]\right\} .
\end{aligned}
$$

Der letzte Ausdruck in (6) ist die Zusammenfassung der beiden Glieder, die durch Vertauschung von $\mathfrak{i}$ und $\mathfrak{m}$ auseinander hervorgehen. Schreiben wir sie beide explizit an, dann muß ein Faktor $\frac{1}{2}$ noch vor die Klammer gesetzt werden. Wir haben jetzt nur zu beachten, $\mathrm{da} \beta \boldsymbol{A}_{\mathrm{mi}}=\boldsymbol{A}_{\text {im }}$ ist, dann kommen wir zu dem erwünschten quadratischen Glied in (1)

$$
\begin{aligned}
\frac{1}{4} \sum_{\mathrm{i}, \mathrm{m}}^{\prime}\left[\vec{\xi}_{\mathrm{i}}-\vec{\xi}_{\mathrm{m}}\right] \cdot \boldsymbol{A}_{\mathrm{im}} \cdot\left[\vec{\xi}_{\mathrm{m}}-\vec{\xi}_{\mathrm{i}}\right] \\
=\frac{1}{2} \sum_{\mathfrak{i}} \vec{\xi}_{\mathfrak{i}} \cdot\left[\mathfrak{f}_{\mathfrak{i}}\left(\vec{\xi}_{\mathfrak{t}}\right)-\sum_{\mathrm{m}} \mathfrak{f}_{\mathfrak{i m}}^{\mathrm{h}}\left(\vec{\xi}_{\mathrm{m}}-\vec{\xi}_{\mathfrak{i}}\right)\right] .
\end{aligned}
$$

Setzen wir ein, so erhalten wir schließlich für die Verzerrungsenergie

$$
\begin{aligned}
\Delta U=U-\frac{1}{2} \sum_{\mathrm{i}, \mathrm{m}}^{\prime} P_{\mathrm{im}}^{0}=\frac{1}{2} \sum_{\mathrm{i}} \vec{\xi}_{\mathrm{i}} \cdot\left[\mathfrak{f}_{\mathfrak{i}}\left(\vec{\xi}_{\mathfrak{f}}\right)\right. \\
\left.-\sum_{\mathrm{m}} \mathfrak{f}_{\mathrm{im}}^{\mathrm{h}}\left(\vec{\xi}_{\mathrm{m}}-\vec{\xi}_{\mathrm{i}}\right)\right]+\frac{1}{2} \sum_{\mathrm{i}, \mathrm{m}} P_{\mathrm{im}}^{\mathrm{h}}\left(\vec{\xi}_{\mathrm{m}}-\vec{\xi}_{\mathrm{i}}\right) .
\end{aligned}
$$

Dies ist die Ausgangsform für alle Energieberechnungen in einem durch nulldimensionale Störungen verzerrten Gitter. Die rechnerische Bedeutung dieser Umformung auf (8) erkennt man dann, wenn man bedenkt, daß die in (4) auftretenden Kräfte und nichtlinearen Ausdrücke bei nulldimensionalen Störungen stets auf kleine Bereiche im Kristall lokalisiert sind, und andererseits die quadratischen Glieder $\frac{1}{2}\left[\vec{\xi}_{\mathfrak{i}}-\vec{\xi}_{\mathfrak{m}}\right] \cdot \boldsymbol{A}_{\mathrm{im}} \cdot\left[\vec{\xi}_{\mathfrak{m}}-\vec{\xi}_{\mathfrak{i}}\right]$ die Energie des elastischen Fernfelds der Störung ausmachen. Genau dieser sich weit in den Kristall erstreckende Anteil der Deformationsenergie wird aber hier zufolge seines Gleichgewichts (7) mit den äußeren und nichtlinea-

(6) ist dies bei den äußeren Kräften $\mathfrak{k}$ sicher auch statthaft. Auf der rechten Seite von (6) treten aber dann zwei unabhängige Summationen über $m$ auf, die nicht in eine zusammengefaßt werden können und daher das Problem nur komplizierter machen würden. 
ren Kräften aus (1) eliminiert. Es genügt somit zur Berechnung der Verzerrungsenergie die Kenntnis der wenigen äußeren Kräfte und der Auslenkungen ihrer Angriffspunkte.

\section{§ 2. Verzerrungsenergie bei Vorverschiebungen}

Wir kommen nun zu Störungen, die sich eindimensional ausdehnen. Hier ist es meist zweckmäßig, nicht von der Ausgangskonfiguration eines idealen Kristalls, sondern von vorverschobenen Lagen auszugehen. (II) befaßt sich ausführlich mit diesem Problem. Die vorverschobenen Lagen werden als bekannte Größen in die gitterstatischen Rechnungen eingeführt. Wir entnehmen sie zum Beispiel vorhergehenden elastizitätstheoretischen Rechnungen, doch gibt es auch andere Möglichkeiten, die nur jeweils so geartet sein müssen, daß zwischen vorverschobenem Kristall und Gleichgewichtskonfiguration kein Potentialpaß mehr überschritten werden muß. Ehe wir uns einem speziellen Modell zuwenden, wollen wir die Energiebestimmung bei vorverschobenen Lagen, soweit es möglich ist, vom allgemeinen Standpunkt aus betrachten. Da nun zur Berechnung der Gleichgewichtslagen zuerst eine Transformation

$$
\vec{\xi}_{\mathfrak{m}}=\mathfrak{v}_{\mathfrak{m}}+\vec{\zeta}_{\mathfrak{m}}
$$

der Freiheitsgrade durchgeführt werden muß, formulieren sich die Gleichgewichtsbedingungen jetzt ausschließlich in den neuen Freiheitsgraden $\vec{\zeta}_{\mathfrak{m}}$, also tritt an die Stelle von (4), nach (II) § 8, das System

$$
\boldsymbol{A}_{\mathfrak{i m}} \cdot\left[\vec{\zeta}_{\mathfrak{m}}-\vec{\zeta}_{\mathfrak{i}}\right]=\mathfrak{F}_{\mathfrak{i}}^{\mathfrak{v}}\left(\vec{\zeta}_{\mathfrak{t}}\right)-\sum_{\mathfrak{m}} \mathfrak{f}_{\mathfrak{i m}}^{\mathrm{hv}}\left(\vec{\zeta}_{\mathfrak{m}}-\vec{\zeta}_{\mathfrak{i}}\right)
$$

$\mathfrak{f}_{\mathfrak{i}}^{\mathfrak{v}}\left(\overrightarrow{\zeta_{\mathfrak{f}}}\right)$ ist diesmal nicht identisch mit den äußeren Kräften, sondern enthält außer diesen noch Zwangskräfte, die von der Deformation durch die Vorverschiebungen herrühren. Ihre spezielle Gestalt werden wir noch herleiten. $\mathfrak{f}_{i \mathrm{~m}}^{\text {hv }}\left(\vec{\zeta}_{\mathrm{m}}-\vec{\zeta}_{\mathrm{i}}\right)$ sind wieder die höheren Glieder in Abhängigkeit von $\left(\vec{\zeta}_{\mathrm{m}}-\vec{\zeta}_{\mathrm{i}}\right)$.

Um (10) in analoger Weise zum nulldimensionalen Problem für die Reduktion des Energieausdrucks benützen zu können, muß der Energieaus- druck selbst auf die neuen Freiheitsgrade umgeschrieben werden

$$
U=\frac{1}{2} \sum_{i, m}^{\prime} P_{i m}\left(\mathfrak{v}_{\mathfrak{m}}-\mathfrak{v}_{\mathfrak{i}}+\vec{\zeta}_{\mathrm{m}}-\vec{\zeta}_{\mathrm{i}}\right) .
$$

Die Einführung der Vorverschiebungen $\mathfrak{b}_{m}$ erfordert jetzt die Unterscheidung von zwei Fällen:

I. In derjenigen Umgebung eines Punktes $i$, in der wir die Wechselwirkung mit den Gitterbausteinen $m$ nicht vernachlässigen dürfen, erreichen die Beträge der Verschiebungsdifferenzen $\mathfrak{b}_{m}-\mathfrak{b}_{i}$ in keinem Fall den Betrag eines Translationsvektors de $e_{j}$. Oder etwas allgemeiner: Wir wollen fordern, daß

$$
\begin{gathered}
\mathfrak{v}_{\mathrm{m}}-\mathfrak{v}_{\mathfrak{i}}=a \mathfrak{e}_{1} d_{1}+b \mathfrak{e}_{2} d_{2}+c \mathfrak{e}_{3} d_{3} \\
\text { mit }|a|,|b|,|c|<1
\end{gathered}
$$

ist. Dann ist bei einer Entwicklung von (11) um die ursprünglichen Ideallagen die Konvergenz noch gesichert.

II. In der Wechselwirkungsumgebung von $i$ gibt es neben Gitterpunkten $\mathrm{m}^{\prime}$, welche die Bedingung (12) erfüllen, auch Punkte $m^{\prime \prime}$, für die die Vorverschiebungsdifferenzen den Wert eines Translationsvektors überschreiten, eventuell sogar mehrfach. Oder wieder allgemeiner formuliert: Es soll

$$
\begin{gathered}
\mathfrak{v}_{\mathrm{m} \prime}-\mathfrak{v}_{\mathrm{i}}=a \mathfrak{e}_{1} d_{1}+b \mathfrak{e}_{2} d_{2}+c \mathfrak{e}_{3} d_{3} \\
\text { mit }|a|,|b|,|c| \geq 1
\end{gathered}
$$

sein. In diesem Fall kann zum Beispiel durch die Vorverschiebungen die plastische Deformation eines Teilbereichs des Kristalls beschrieben werden.

Da wir bei der Berechnung einer gestörten Kristallstruktur mit Kraftgleichungen der Gestalt (10) arbeiten, die aus der Entwicklung der Potentiale nach den Auslenkungsdifferenzen $\left(\vec{\zeta}_{\mathrm{m}}-\vec{\zeta}_{\mathrm{i}}\right)$ hervorgehen, müssen wir zuerst die Auswirkung einer solchen plastischen Deformation auf die Energie in der impliziten Form (11) untersuchen.

Wir beschränken uns in diesem Paragraphen jedoch vorerst auf den Fall, daß für alle Punkte $i$ des Kristalls die Forderung (12) erfüllt sei. Dann können wir unbedenklich entwickeln und die einzelnen Potentiale für die Punkte $i$ summieren. Wir erhalten

$$
\begin{aligned}
\Delta U=U-\frac{1}{2} \sum_{\mathrm{i}, \mathrm{m}}^{\prime} P_{\mathrm{im}}^{0}=\frac{1}{2} \sum_{\mathrm{i}, \mathrm{m}}^{\prime \prime}\left\{\frac{1}{2}\left[\mathfrak{v}_{\mathrm{i}}-\mathfrak{v}_{\mathrm{m}}\right] \cdot \boldsymbol{A}_{\mathrm{im}} \cdot\left[\mathfrak{v}_{\mathrm{m}}-\mathfrak{v}_{\mathrm{i}}\right]+\left[\mathfrak{v}_{\mathrm{i}}-\mathfrak{v}_{\mathrm{m}}\right] \cdot \boldsymbol{A}_{\mathrm{im}} \cdot\left[\vec{\zeta}_{\mathrm{m}}-\vec{\zeta}_{\mathrm{i}}\right]\right. \\
\left.+\frac{1}{2}\left[\vec{\zeta}_{\mathrm{i}}-\vec{\zeta}_{\mathrm{m}}\right] \cdot \boldsymbol{A}_{\mathrm{im}} \cdot\left[\vec{\zeta}_{\mathrm{m}}-\vec{\zeta}_{\mathrm{i}}\right]+P_{\mathrm{im}}^{\mathrm{h}}\left(\mathfrak{v}_{\mathrm{m}}-\mathfrak{v}_{\mathrm{i}}+\vec{\zeta}_{\mathrm{m}}-\vec{\zeta}_{\mathrm{i}}\right)\right\} .
\end{aligned}
$$

Das dritte Glied in (14) entspricht jetzt dem quadratischen Glied in (2) und kann in analoger Weise zu § 1 
durch die Kräfte (10) ersetzt werden. Die reduzierte Form von (14) lautet dann

$$
\begin{aligned}
& \Delta U=\frac{1}{2} \sum_{i, m}^{\prime}\left\{\frac{1}{2}\left[\mathfrak{v}_{\mathrm{i}}-\mathfrak{v}_{\mathrm{m}}\right] \cdot \boldsymbol{A}_{\mathrm{im}} \cdot\left[\mathfrak{v}_{\mathrm{m}}-\mathfrak{v}_{\mathrm{i}}\right]+\left[\mathfrak{v}_{\mathrm{i}}-\mathfrak{v}_{\mathrm{m}}\right] \cdot \boldsymbol{A}_{\mathrm{im}} \cdot\left[\vec{\zeta}_{\mathrm{m}}-\vec{\zeta}_{\mathrm{i}}\right]+P_{\mathfrak{i m}}^{\mathrm{h}}\left(\mathfrak{v}_{\mathrm{m}}-\mathfrak{v}_{\mathrm{i}}+\vec{\zeta}_{\mathrm{m}}-\vec{\zeta}_{\mathrm{i}}\right)\right\} \\
& +\frac{1}{2} \sum_{i} \vec{\zeta}_{i} \cdot\left[\mathfrak{f}_{\mathfrak{i}}^{\mathfrak{v}}\left(\vec{\zeta}_{\mathfrak{z}}\right)-\sum_{\mathrm{m}} \mathfrak{f}_{\mathfrak{i} \mathrm{m}}^{\mathrm{hv}}\left(\vec{\zeta}_{\mathrm{m}}-\vec{\zeta}_{\mathfrak{i}}\right)\right]
\end{aligned}
$$

Wir wollen die Kräfte $\mathfrak{f}_{\mathfrak{i}}^{\mathfrak{p}}$ und $\mathfrak{f}_{\mathfrak{i} \mathrm{m}}^{\text {hv }}$ noch etwas näher betrachten. Dazu differenzieren wir (14) und finden daraus die Gleichgewichtsbedingungen bei vorverschobenen Lagen zu

$$
\boldsymbol{A}_{\mathrm{im}} \cdot\left[\vec{\zeta}_{\mathrm{m}}-\vec{\zeta}_{\mathrm{i}}\right]=\mathfrak{f}_{\mathrm{i}}\left(\vec{\zeta}_{\mathfrak{t}}\right)-\sum_{\mathrm{m}} \boldsymbol{A}_{\mathrm{im}} \cdot\left[\mathfrak{v}_{\mathrm{m}}-\mathfrak{v}_{\mathrm{i}}\right]-\sum_{\mathrm{m}} \mathfrak{f}_{\mathrm{im}}^{\mathrm{h}}\left(\mathfrak{v}_{\mathrm{m}}-\mathfrak{v}_{\mathfrak{i}}+\vec{\zeta}_{\mathrm{m}}-\vec{\zeta}_{\mathfrak{i}}\right) .
$$

Durch Vergleich mit (10) finden wir dann

$$
\mathfrak{f}_{\mathfrak{i}}^{\mathfrak{v}}\left(\overrightarrow{\zeta_{\mathfrak{t}}}\right)=\mathfrak{f}_{\mathfrak{i}}\left(\overrightarrow{\zeta_{\mathfrak{t}}}\right)-\sum_{\mathrm{m}} \boldsymbol{A}_{\mathfrak{i} \mathrm{m}} \cdot\left[\mathfrak{v}_{\mathrm{m}}-\mathfrak{v}_{\mathfrak{i}}\right], \quad \mathfrak{f}_{\mathfrak{i}}^{\mathrm{hn}}\left(\vec{\zeta}_{\mathfrak{t}}\right)=\mathfrak{f}_{\mathfrak{i m}}^{\mathrm{h}}\left(\mathfrak{v}_{\mathrm{m}}-\mathfrak{v}_{\mathfrak{i}}+\zeta_{\mathrm{m}}-\zeta_{\mathfrak{i}}\right)
$$

Aus (17 a) ersehen wir jetzt die Gestalt der Zwangskräfte, die zu den äußeren Kräften bei vorverschobenen Lagen noch hinzutreten. (17 a) und $(17 \mathrm{~b})$ in (15) eingeführt ergibt schließlich:

$$
\begin{aligned}
\Delta U=\frac{1}{2} \sum_{\mathrm{i}, \mathrm{m}}^{\prime}\left\{\frac { 1 } { 2 } [ \mathfrak { v } _ { \mathfrak { i } } - \mathfrak { v } _ { \mathrm { m } } ] \cdot \boldsymbol { A } _ { \mathrm { im } } \cdot \left[\mathfrak{v}_{\mathrm{m},}\right.\right. & \left.-\mathfrak{v}_{\mathrm{i}}\right]+\frac{1}{2}\left[\mathfrak{v}_{\mathrm{i}}-\mathfrak{v}_{\mathrm{m}}\right] \cdot \boldsymbol{A}_{\mathfrak{i m}} \cdot\left[\vec{\zeta}_{\mathrm{m}}-\vec{\zeta}_{\mathrm{i}}\right] \\
& \left.+P_{\mathrm{im}_{\mathrm{m}}}^{\mathrm{h}}\left(\mathfrak{v}_{\mathrm{m}}-\mathfrak{v}_{\mathrm{i}}+\vec{\zeta}_{\mathrm{m}}-\vec{\zeta}_{\mathrm{i}}\right)\right\}+\frac{1}{2} \sum_{\mathrm{i}} \vec{\zeta}_{\mathrm{i}} \cdot\left[\mathfrak{f}_{\mathrm{i}}\left(\vec{\zeta}_{\mathrm{f}}\right)-\sum_{\mathrm{m}} \mathfrak{f}_{\mathrm{im}}^{\mathrm{h}}\left(\mathfrak{v}_{\mathrm{m}}-\mathfrak{v}_{\mathrm{i}}+\vec{\zeta}_{\mathrm{m}}-\vec{\zeta}_{\mathrm{i}}\right)\right] .
\end{aligned}
$$

\section{§ 3. Verzerrungsenergie bei plastischen Verformungen}

Wir kommen nun zum zweiten Fall. Wie schon erwähnt, zeichnet er sich dadurch aus, daß in der Wechselwirkungsumgebung des Punktes $i$ nicht nur Punkte $\mathfrak{m}^{\prime}$ enthalten sind, für deren Vorverschiebungsdifferenzen (12) gilt, sondern auch solche Punkte $\mathfrak{m}^{\prime \prime}$, deren Vorverschiebungsdifferenzen $\mathfrak{b}_{\mathfrak{m}}-\mathfrak{b}_{\mathfrak{i}}(13)$ gehorchen. Es ist also eine Aufspaltung der Potentiale $P_{\mathrm{im}}$ entsprechend der zu unterscheidenden Teilbereiche unumgänglich.

Greifen wir einen beliebigen Punkt $i$ heraus, so ist seine Energie im Potential der umgebenden Ionen ${ }^{5}$

$$
\begin{aligned}
& U_{\mathrm{i}}=\sum_{\mathrm{m}} P_{\mathrm{im}}\left(\mathfrak{v}_{\mathrm{m}}-\mathfrak{v}_{\mathrm{i}}+\vec{\zeta}_{\mathrm{m}}-\vec{\zeta}_{\mathrm{i}}\right) \\
& =\sum_{\mathrm{m}^{\prime}} P_{\mathrm{im}}\left(\mathfrak{v}_{\mathrm{m}}-\mathfrak{v}_{\mathrm{i}}+\vec{\zeta}_{\mathrm{m}}-\vec{\zeta}_{\mathfrak{i}}\right) \\
& +\sum_{\mathrm{m}^{\prime \prime}} P_{\mathrm{i} \mathrm{m}}\left(\mathfrak{b}_{\mathrm{m}}-\mathfrak{v}_{\mathrm{i}}+\vec{\zeta}_{\mathrm{m}}-\vec{\zeta}_{\mathrm{i}}\right) \text {. }
\end{aligned}
$$

Die Aufspaltung in zwei Teilbereiche ist jetzt durch die Unterscheidung der beiden Summationen über $\mathfrak{m}^{\prime}$ bzw. $\mathfrak{m}^{\prime \prime}$ vollzogen. Die erstere erfasse alle diejenigen Wechselwirkungen, die Fall I gehorchen. Wir können diese Potentiale ohne weiteres nach dem Vorbild von $\S 2$ entwickeln. Ein Entwicklung der anderen Potentiale kommt dagegen erst in Frage, wenn

5 Die Gesamtenergie des Kristalls baut sich dann zusammen aus $U=\frac{1}{2} \sum_{i} U_{i}$. wir sie einem Translationstheorem unterworfen haben. Dieses Theorem wurde in (II), § 2, ausführlich erörtert. Wir wollen es hier noch etwas allgemeiner fassen und dazu annehmen, die Verschiebungsdifferenzen seien mit bestimmten Gittervektoren ${ }^{6}$ $e_{m}=\alpha_{m} e_{1}+\beta_{m} e_{2}+\gamma_{m} e_{3}$ verknüpft durch die Beziehung

$$
\mathfrak{v}_{\mathfrak{m}}-\mathfrak{v}_{\mathfrak{i}}+\mathfrak{w}_{\mathrm{m}}-\mathfrak{w}_{\mathfrak{i}}=\mathfrak{e}_{\mathrm{m}} d,
$$

wobei $\mathfrak{w}_{\mathfrak{m}}-\mathfrak{w}_{\mathfrak{i}}$ einen Ergänzungsvektor bedeutet, der den Forderungen von Fall I gehorcht. Durch die Auswahl eines bestimmten Satzes ganzer Zahlen $\alpha_{\mathrm{m}}, \beta_{\mathrm{m}}, \gamma_{\mathrm{m}}$ läßt sich dies immer erreichen. Ersetzen wir im letzten Glied von (19) die Vorverschiebungsdifferenzen mit Hilfe von (20) durch die entsprechenden Gitter- und Ergänzungsvektoren, so können wir jetzt die angekündigte Translation durchführen. Es ist nämlich

$$
\begin{aligned}
\sum_{\mathfrak{m}^{\prime \prime}} P_{\mathfrak{i m}}\left(\mathfrak{v}_{\mathrm{m}}-\right. & \left.\mathfrak{y}_{\mathrm{i}}+\vec{\zeta}_{\mathrm{m}}-\vec{\zeta}_{\mathfrak{i}}\right) \\
& =\sum_{\mathfrak{m}^{\prime \prime}} P_{\mathfrak{i} \mathrm{m}}\left(d e_{\mathrm{m}}-\left(\mathfrak{w}_{\mathfrak{m}}-\mathfrak{w}_{\mathfrak{i}}\right)+\vec{\zeta}_{\mathrm{m}}-\vec{\zeta}_{\mathfrak{i}}\right) \\
& =\sum_{\mathfrak{m}^{\prime \prime}} P_{\mathrm{i}, \mathrm{m}}+\mathfrak{e}_{\mathrm{m}}\left(-\mathfrak{w}_{\mathrm{m}}+\mathfrak{w}_{\mathfrak{i}}+\vec{\zeta}_{\mathrm{m}}-\vec{\zeta}_{\mathfrak{i}}\right) .
\end{aligned}
$$

Nach den Erläuterungen von (II), § 2, steht jetzt der Entwicklung der Potentiale (21) um die neuen Entwicklungsorte $\left|\Re_{\mathrm{m}+\mathrm{e}_{\mathrm{m}}}^{0}-\Re_{\mathrm{i}}^{0}\right|$ nichts mehr im Wege. Wir finden für $\Delta U_{\mathrm{i}}$

${ }^{6} a_{\mathfrak{m}}, \beta_{\mathrm{m}}, \gamma_{\mathrm{m}}$ sind ganze Zahlen. Der Einfachheit halber schreiben wir $\mathfrak{e}^{\mathrm{m}}$ an Stelle von $\mathfrak{e}\left(\alpha_{\mathfrak{m}}, \beta_{\mathrm{m}}, \gamma_{\mathrm{m}}\right)$ und nehmen nur ein kubisches Gitter mit der Gitterkonstanten $d$ an. 


$$
\begin{aligned}
& \Delta U_{\mathrm{i}}=U_{\mathrm{i}}-\sum_{\mathrm{m}^{\prime}} P_{\mathrm{im}}^{0}-\sum_{\mathrm{m}^{\prime \prime}} P_{\mathrm{i} \mathrm{m}}^{0} \\
& =\sum_{\mathrm{m}}\left\{\Omega_{\mathrm{im}} \cdot\left[\mathfrak{v}_{\mathrm{i}}-\mathfrak{v}_{\mathrm{m}}+\vec{\zeta}_{\mathrm{i}}-\vec{\zeta}_{\mathrm{m}}\right]+\frac{1}{2}\left[\mathfrak{v}_{\mathrm{i}}-\mathfrak{v}_{\mathrm{m}}+\vec{\zeta}_{\mathrm{i}}-\vec{\zeta}_{\mathrm{m}}\right] \cdot \boldsymbol{A}_{\mathrm{im}} \cdot\left[\mathfrak{v}_{\mathrm{m}}-\mathfrak{v}_{\mathrm{i}}+\vec{\zeta}_{\mathrm{m}}-\vec{\zeta}_{\mathrm{i}}\right]+P_{\mathrm{im}}^{\mathrm{h}}\left(\mathfrak{v}_{\mathrm{m}}-\mathfrak{v}_{\mathrm{i}}+\vec{\zeta}_{\mathrm{m}}-\vec{\zeta}_{\mathrm{i}}\right)\right. \\
& +\sum_{m^{\prime \prime}}\left\{\mathfrak{R}_{i m}+\mathfrak{e}_{m} \cdot\left[-\mathfrak{w}_{i}+\mathfrak{w}_{m}+\vec{\zeta}_{i}-\vec{\zeta}_{m}\right]+\frac{1}{2}\left[-\mathfrak{w}_{i}+\mathfrak{w}_{m}+\vec{\zeta}_{i}-\vec{\zeta}_{m}\right] \cdot \boldsymbol{A}_{i m}+\mathfrak{e}_{m} \cdot\left[-\mathfrak{w}_{m}+\mathfrak{w}_{i}+\vec{\zeta}_{m}-\vec{\zeta}_{i}\right]\right. \\
& \left.+P_{\mathrm{im}+\mathfrak{e}_{\mathrm{m}}}^{\mathrm{h}}\left(-\mathfrak{w}_{\mathrm{m}}+\mathfrak{w}_{\mathrm{i}}+\vec{\zeta}_{\mathrm{m}}-\vec{\zeta}_{\mathrm{i}}\right)\right\} .
\end{aligned}
$$

Von Interesse ist, daß bei dieser allgemeinsten mathematischen Formulierung auch bei einer nachfolgenden Summation über $i$ die linearen Glieder keineswegs verschwinden müssen. Wir können nämlich nicht erwarten, daß nach Ausführung der Translation (21) die Entwicklungsorte wieder vollständig und lückenlos auf ein ideal angeordnetes System abgebildet worden sind. Wir erkennen hier schon, daß ohne einschneidende Beschränkungen keine einfacheren Aussagen mehr erwartet werden können.

Noch von einem anderen Gesichtspunkt aus ist eine ganz allgemeine Formulierung der Energie außerordentlich kompliziert. Bedenken wir etwa, daß für einen zweiten Punkt $i^{\prime}$ die Einteilung in $\mathfrak{m}^{\prime}$ und $\mathfrak{m}^{\prime \prime}$ keineswegs mehr zusammenfallen muß mit der Einteilung, wie sie für den Punkt $i$ gegeben war, so sehen wir, daß es für die praktische Anwendung wenig Sinn hat, alle die voneinander abweichenden Einteilungen bei einer Summation über $\mathrm{i}$ zu unterscheiden.

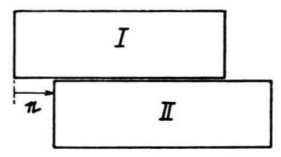

Abb. 1.

Wir beschränken uns daher bei allen weiteren Erörterungen dieses Paragraphen auf das physikalisch sinnvolle Beispiel einer plastischen Verformung entlang einer Gleitebene. Eine solche plastische Verformung können wir zum Beispiel erreichen, indem wir allen Gitterbausteinen einer der beiden Kristallhälften von Abb. 1 eine gemeinsame gleichgroße Vorverschiebung erteilen, die größer oder mindestens gleich einem Atomabstand ist. Wir lösen uns jetzt von der obigen Einteilung der Punkte in $\mathfrak{m}^{\prime}$ und $\mathrm{m}^{\prime \prime}$, und benennen hier die Gitter- bausteinen der Kristallhälfte I in Abb. 1 mit m', die der Kristallhälfte II mit m". Dann brauchen wir keine Fallunterscheidung mehr für die herausgegriffenen Punkte i machen.

Natürlich ist eine Abgleitung, die entlang der gesamten Gleitebene stattgefunden hat, verhältnismäßig uninteressant. Doch wollen wir im Augenblick von Versetzungen noch absehen und nur annehmen, daß neben plastischen auch elastische Deformationen vorliegen. Es ist für dieses Problem sinnvoll, drei Einschränkungen zu fordern:

1) In (20) sei $\mathfrak{e}^{\prime}=0$ und $\mathfrak{e}$ m:" unabhängig von $\mathfrak{m}^{\prime \prime}$, also $\mathrm{e}_{\mathrm{m}} \mathrm{m}^{\prime}=\mathfrak{e}$.

2) $e$ liege in der Gleitebene. Damit ist bei beliebig ausgedehntem Kristall nach Ausübung der Vorverschiebung wieder eine Abbildung der Entwicklungsorte auf ein Idealgitter gewährleistet.

3) Aus den Gittergleichungen für das Problem soll

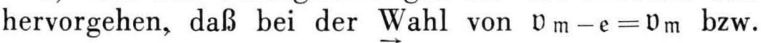
$\mathfrak{w}_{\mathfrak{m}-\mathfrak{e}}=\mathfrak{w}_{\mathfrak{m}}$ auch $\zeta_{\mathfrak{m}-\mathfrak{e}}=\vec{\zeta}_{\mathfrak{m}}$ werden muß. Dies läßt sich eventuell aus Symmetriebetrachtungen ableiten und bedingt eine Translationssymmetrie des Problems entlang $e$.

Die Forderung 3) ist recht einschneidend, vereinfacht aber die folgenden Betrachtungen außerordentlich ${ }^{7}$.

Wählen wir jetzt zum Beispiel einen Punkt $i$ im Kristallbereich I, so lautet für diesen die potentielle Energie (19) bei Berücksichtigung der Forderungen 1) und 2)

$$
\begin{aligned}
U_{\mathfrak{i}^{\prime}}=\sum_{\mathfrak{m}^{\prime}} P_{\mathfrak{i}^{\prime} \mathfrak{m}}\left(\mathfrak{v}_{\mathfrak{m}}-\mathfrak{v}_{\mathfrak{i}^{\prime}}+\vec{\zeta}_{\mathfrak{m}}-\vec{\zeta}_{\mathfrak{i}^{\prime}}\right) \\
+\sum_{\mathfrak{m}^{\prime \prime}} P_{\mathfrak{i}^{\prime}, \mathfrak{m}+\mathfrak{e}}\left(-\mathfrak{w}+\mathfrak{w}_{\mathfrak{i}^{\prime}}+\vec{\zeta}_{\mathfrak{m}}-\vec{\zeta}_{\mathfrak{i}^{\prime}}\right) .
\end{aligned}
$$

Mit Hilfe der Einschränkung 3) läßt sich die zweite Summe in (23) noch erheblich vereinfachen:

$$
\begin{array}{r}
\sum_{\mathfrak{m}^{\prime \prime}} P_{\mathfrak{i}^{\prime}, \mathfrak{i}+\mathfrak{e}}\left(-\mathfrak{w}_{\mathfrak{m}}+\mathfrak{w}_{\mathfrak{i}^{\prime}}+\vec{\zeta}_{\mathfrak{m}}-\vec{\zeta}_{\mathfrak{i}^{\prime}}\right)=\sum_{\mathfrak{m}^{\prime \prime}-\mathfrak{e}} P_{\mathfrak{i}^{\prime} \mathfrak{m}}\left(-\mathfrak{w}_{\mathfrak{m}-\mathfrak{e}}+\mathfrak{w}_{\mathfrak{i}^{\prime}}+\vec{\zeta}_{\mathfrak{m}-\mathfrak{e}}-\vec{\zeta}_{\mathfrak{i}^{\prime}}\right) \\
=\sum_{\mathfrak{m}^{\prime \prime}} P_{\mathfrak{i}^{\prime} \mathfrak{m}}\left(-\mathfrak{w}_{\mathfrak{m}}+\mathfrak{w}_{\mathfrak{i}^{\prime}}+\vec{\zeta}_{\mathfrak{m}}-\vec{\zeta}_{\mathfrak{i}^{\prime}}\right) .
\end{array}
$$

Eine entsprechende Gleichung gilt für einen Punkt $i^{\prime \prime}$ im Kristallbereich II.

Wir sind damit in der Lage, die Gesamtenergie des Kristalls anzugeben. Sie setzt sich aus vier Anteilen zusammen:

$$
\begin{aligned}
& U=\frac{1}{2} \sum_{i} U_{\mathfrak{i}}=\frac{1}{2} \sum_{i^{\prime}} \sum_{\mathfrak{m}^{\prime}} P_{\mathfrak{i}_{\mathrm{m}}}\left(\mathfrak{v}_{\mathrm{m}}-\mathfrak{v}_{\mathfrak{i}}+\vec{\zeta}_{\mathrm{m}}-\vec{\zeta}_{\mathfrak{i}}\right)+\frac{1}{2} \sum_{\mathrm{i}^{\prime}} \sum_{\mathrm{m}^{\prime \prime}} P_{\mathfrak{i} \mathrm{m}}\left(-\mathfrak{w}_{\mathrm{m}}+\mathfrak{w}_{\mathfrak{i}}+\vec{\zeta}_{\mathrm{m}}-\vec{\zeta}_{\mathrm{i}}\right) \\
& +\frac{1}{2} \sum_{i^{\prime \prime}} \sum_{\mathfrak{m}^{\prime}} P_{\mathrm{im}}\left(-\mathfrak{w}_{\mathrm{m}}+\mathfrak{w}_{\mathrm{i}}+\vec{\zeta}_{\mathrm{m}}-\vec{\zeta}_{\mathrm{i}}\right)+\frac{1}{2} \sum_{\mathrm{i}^{\prime \prime}} \sum_{\mathrm{m}^{\prime \prime}} P_{\mathrm{im}}\left(\mathfrak{v}_{\mathrm{m}}-\mathfrak{v}_{\mathfrak{i}}+\vec{\zeta}_{\mathrm{m}}-\vec{\zeta}_{\mathfrak{i}}\right) .
\end{aligned}
$$

${ }^{7}$ Forderung 3) ist zum Beispiel bei der Schraubenversetzung erfüllt. 
Es ist nun nicht weiter schwierig, die Entwicklung von (25) auszuführen. Mit den Kräften, die wir aus einer solchèn Entwicklung durch Differentiation erhalten, ist die Reduktion der Energiegleichung genau so möglich wie in $\S 2$.

Für einen Punkt $i{ }^{\prime}$ finden wir die Kraftgleichung zu

$$
\begin{aligned}
\sum_{m} \boldsymbol{A}_{\mathfrak{i}^{\prime} \mathfrak{m}} \cdot\left[\vec{\zeta}_{\mathfrak{m}}-\vec{\zeta}_{\mathfrak{i}^{\prime}}\right]=-\sum_{\mathfrak{m}^{\prime}}\left\{\boldsymbol{A}_{\mathfrak{i}^{\prime} \mathfrak{m}} \cdot\left[\mathfrak{v}_{\mathfrak{m}}-\mathfrak{v}_{\mathfrak{i}^{\prime}}\right]+\mathfrak{f}_{\mathfrak{i}^{\prime} \mathfrak{m}}^{h}\left(\mathfrak{v}_{\mathfrak{m}}-\mathfrak{v}_{\mathfrak{i}^{\prime}}+\vec{\zeta}_{\mathfrak{m}}-\vec{\zeta}_{\mathfrak{i}^{\prime}}\right)\right\} \\
\quad-\sum_{\mathfrak{m}^{\prime \prime}}\left\{-\boldsymbol{A}_{\mathfrak{i}^{\prime} \mathfrak{m}} \cdot\left[\mathfrak{w}_{\mathfrak{m}}-\mathfrak{w}_{\mathfrak{i}^{\prime}}\right]+\mathfrak{f}_{\mathfrak{i}^{\prime} \mathfrak{m}}^{\mathrm{h}}\left(-\mathfrak{w}_{\mathfrak{m}}+\mathfrak{w}_{\mathfrak{i}^{\prime}}+\vec{\zeta}_{\mathfrak{m}}-\vec{\zeta}_{\mathfrak{i}^{\prime}}\right)\right\} .
\end{aligned}
$$

Eine entsprechende Gleichung gilt wieder für i". Der linken Seite von (26) bzw. ihrer entsprechenden Gleichung für $i^{\prime \prime}$ sieht man die Vergangenheit der Vorverschiebung nicht an. Wir können also wie üblich mit $\frac{1}{2} \vec{\zeta}_{i^{\prime}}$ bzw. $\frac{1}{2} \overrightarrow{\zeta_{i}^{\prime \prime}}$ multiplizieren und die über alle $i$ summierten Glieder $\frac{1}{2} \sum_{i, \mathrm{~m}} \overrightarrow{\zeta_{i}} \cdot \boldsymbol{A}_{\mathrm{im}} \cdot\left[\overrightarrow{\zeta_{\mathrm{m}}}-\overrightarrow{\zeta_{\mathrm{i}}}\right]$ so umordnen, daß das quadratische Glied $\frac{1}{4} \sum_{\mathrm{i}, \mathrm{m}}\left[\vec{\zeta}_{\mathrm{i}}-\vec{\zeta}_{\mathrm{m}}\right] \cdot \boldsymbol{A}_{\mathrm{im}} \cdot\left[\vec{\zeta}_{\mathrm{m}}-\vec{\zeta}_{\mathrm{i}}\right]$ wieder durch die Kraftgleichungen ersetzt werden kann.

\section{§ 4. Verzerrungsenergie bei fehlenden äußeren Kräften}

Bislang schrieben wir zwar die Energie eines herausgegriffenen und festgehaltenen Punktes in Form der unentwickelten Potentiale an, doch waren wir für die Reduktion mit Hilfe der Kraftgleichung auf eine Summation von $\mathfrak{i}$ über den Gesamtkristall angewiesen. Es ist aber für die nachfolgenden Betrachtungen am speziellen Modell notwendig, auch die reduzierte Form der Energiegleichung für einen einzelnen herausgegriffenen Punkt anzugeben. Wenn wir, was im folgenden möglich ist, keine äußeren Kräfte zu berücksichtigen haben, gelingt dies durch Umordnung der höheren Kraftglieder an Stelle der Umordnung der quadratischen Glieder in (6) bzw. in den entsprechenden Gleichungen mit Vorverschiebungen. Es ist nämlich in (6), (18) und (26) möglich, die höheren Glieder $\mathfrak{f}_{i m}^{\mathrm{h}} \cdot \vec{\zeta}_{\mathrm{i}}$ auf die Differenzen $\left(\vec{\zeta}_{\mathrm{i}}-\vec{\zeta}_{\mathrm{m}}\right)$ umzuschreiben

$$
\frac{1}{2} \sum_{i, m} \mathfrak{f}_{i_{m}}^{\mathrm{h}} \cdot \vec{\zeta}_{\mathrm{i}}=\frac{1}{4} \sum_{\mathrm{i}, \mathrm{m}}\left\{\mathfrak{f}_{\mathrm{im}}^{\mathrm{h}} \cdot \vec{\zeta}_{\mathrm{i}}+\mathfrak{f}_{\mathrm{m} i \mathrm{i}}^{\mathrm{h}} \cdot \vec{\zeta}_{\mathrm{m}}\right\} .
$$

$\mathrm{Da}$ aber allgemein gezeigt werden kann, daß

$$
\mathfrak{f}_{\mathrm{mi}}^{\mathrm{h}}=-\mathfrak{f}_{\mathrm{i} m}^{\mathrm{h}}
$$

ist, erhalten wir schließlich

$$
\frac{1}{2} \sum_{i, m} \mathfrak{f}_{i m}^{h} \cdot \overrightarrow{\zeta_{i}}=\frac{1}{4} \sum_{i, m} \mathfrak{f}_{i m}^{h} \cdot\left[\vec{\zeta}_{i}-\vec{\zeta}_{m}\right] .
$$

Eine Zusammenfassung aller Beiträge für den Punkt $i$ läßt sich jetzt in den Doppelsummen der Gesamtenergie ohne weiteres durchführen. Damit können wir die Energie für den Punkt $i$ auch in reduzierter Form angeben. Sie lautet im Fall I

$$
\begin{aligned}
\Delta U_{\mathrm{i}}=U_{\mathrm{i}}-\sum_{\mathrm{m}} P_{\mathrm{im}}^{0}=\sum_{\mathrm{m}}\left\{\frac{1}{2}\left[\mathfrak{v}_{\mathfrak{i}}-\mathfrak{v}_{\mathrm{m}}\right] \cdot \boldsymbol{A}_{\mathfrak{i m}} \cdot\left[\mathfrak{v}_{\mathrm{m}}-\mathfrak{v}_{\mathrm{i}}\right]+\frac{1}{2}\left[\mathfrak{v}_{\mathfrak{i}}-\mathfrak{v}_{\mathrm{m}}\right] \cdot \boldsymbol{A}_{\mathrm{im}} \cdot\left[\vec{\zeta}_{\mathrm{m}}-\vec{\zeta}_{\mathrm{i}}\right]\right. \\
\left.-\frac{1}{2} \mathfrak{f}_{\mathrm{im}}^{\mathrm{h}}\left(\mathfrak{v}_{\mathrm{m}}-\mathfrak{v}_{\mathrm{i}}+\vec{\zeta}_{\mathrm{m}}-\vec{\zeta}_{\mathfrak{i}}\right) \cdot\left[\vec{\zeta}_{\mathrm{i}}-\vec{\zeta}_{\mathrm{m}}\right]+P_{\mathrm{im}}^{\mathrm{h}}\left(\mathfrak{v}_{\mathrm{m}}-\mathfrak{v}_{\mathrm{i}}+\vec{\zeta}_{\mathrm{m}}-\vec{\zeta}_{\mathfrak{i}}\right)\right\}
\end{aligned}
$$

und im Fall II

$$
\begin{aligned}
& \Delta U_{\mathfrak{i}}=U_{\mathfrak{i}}-\sum_{\mathrm{m}} P_{\mathrm{im}}^{0}=\sum_{\mathrm{m}^{\prime}}\left\{\frac{1}{2}\left[\mathfrak{v}_{\mathfrak{i}}-\mathfrak{v}_{\mathrm{m}}\right] \cdot \boldsymbol{A}_{\mathfrak{i} \mathrm{m}} \cdot\left[\mathfrak{v}_{\mathrm{m}}-\mathfrak{v}_{\mathrm{i}}\right]+\frac{1}{2}\left[\mathfrak{v}_{\mathrm{i}}-\mathfrak{v}_{\mathrm{m}}\right] \cdot \boldsymbol{A}_{\mathrm{im}} \cdot\left[\vec{\zeta}_{\mathrm{m}}-\vec{\zeta}_{\mathrm{i}}\right]\right. \\
& \left.-\frac{1}{2} \mathfrak{f}_{\mathfrak{i}}^{\mathrm{h}}\left(\mathfrak{v}_{\mathrm{m}}-\mathfrak{v}_{\mathfrak{i}}+\vec{\zeta}_{\mathrm{m}}-\vec{\zeta}_{\mathfrak{i}}\right) \cdot\left[\vec{\zeta}_{\mathfrak{i}}-\vec{\zeta}_{\mathrm{m}}\right]+P_{\mathrm{im}}^{\mathrm{h}}\left(\mathfrak{v}_{\mathrm{m}}-\mathfrak{v}_{\mathfrak{i}}+\vec{\zeta}_{\mathrm{m}}-\vec{\zeta}_{\mathfrak{i}}\right)\right\} \\
& +\sum_{\mathfrak{m}^{\prime \prime}}\left\{\frac{1}{2}\left[\mathfrak{w}_{\mathrm{i}}-\mathfrak{w}_{\mathrm{m}}\right] \cdot \boldsymbol{A}_{\mathrm{im}} \cdot\left[\mathfrak{w}_{\mathrm{m}}-\mathfrak{w}_{\mathrm{i}}\right]-\frac{1}{2}\left[\mathfrak{w}_{\mathrm{i}}-\mathfrak{w}_{\mathrm{m}}\right] \cdot \boldsymbol{A}_{\mathfrak{i m}} \cdot\left[\vec{\zeta}_{\mathrm{m}}-\vec{\zeta}_{\mathrm{i}}\right]\right. \\
& \left.-\frac{1}{2} \mathfrak{f}_{\mathfrak{i} m}^{\mathrm{h}}\left(-\mathfrak{w}_{\mathrm{m}}+\mathfrak{w}_{\mathfrak{i}}+\zeta_{\mathrm{m}}-\zeta_{\mathfrak{i}}\right) \cdot\left[\vec{\zeta}_{\mathfrak{i}}-\vec{\zeta}_{\mathrm{m}}\right]+P_{\mathfrak{i} \mathrm{m}}^{\mathrm{h}}\left(-\mathfrak{w}_{\mathrm{m}}+\mathfrak{w}_{\mathfrak{i}}+\vec{\zeta}_{\mathrm{m}}-\vec{\zeta}_{\mathfrak{i}}\right)\right\} .
\end{aligned}
$$

In $(31)$ ist die Einteilung in $\mathrm{m}^{\prime}$ und $\mathrm{m}^{\prime \prime}$ wie in $\S 2$ unter Fall II angegeben.

Es ist leicht einzusehen, daß ohne äußere Kräfte ein Verzerrungszustand des Gitters nur durch gittereigene "Zwangskräfte“ im Gleichgewicht gehalten werden kann. Solche Zwangskräfte treten aber nur bei plastischen Verformungen auf. Bei Versetzungen liegen plastische Verformungen vor. Die Formeln (30) und (31) können wir daher zur Behandlung von speziellen Versetzungsmodellen benützen.

\section{§5. Verzerrungsenergie der Schraubenversetzung}

Wir haben in den vorhergehenden Paragraphen die Theorie der Energieberechnung in der klassischen, nichtlinearen Gitterstatik vom allgemeinen Standpunkt aus abgeleitet. Weitergehende Aussagen können nur noch an speziellen Modellen gemacht werden. Nulldimensionale Störkonfigurationen brauchen, wie erwähnt, keine weiteren Betrachtungen. Dagegen wollen wir in diesem Paragraphen als Repräsentanten einer eindimensiona- 
len Störung, zu deren Berechnung Vorverschiebungen benutzt werden müssen, die Schraubenversetzung untersuchen. Wir können uns weitgehend auf die Ergebnisse von $\S 2$ bis $\S 4$ stützen, die in ihrer Entwicklung schon so angelegt wurden, daß zur Behandlung der Schraubenversetzung keine langwierigen Vorbereitungen mehr notwendig sind.

Da bei der Schraubenversetzung eine plastische Deformation vorliegt, die den drei Forderungen von $\S 3$ gehorcht, können wir für das in (II), §8, untersuchte Modell direkt die Ergebnisse des $\S 3$ verwenden. Bei diesem Modell der Schraubenversetzung liegt die Gleitebene zwischen den Punkten $\mathrm{m}=(m \geqq 0, n, p \geqq 0)$ und den Punkten $m=(m \geqq 0, n, p<0)$. Doch machen wir von ihrer genauen Lage hier keinen Gebrauch. Es genügt die übliche Einteilung in $\mathrm{m}^{\prime}$ und $\mathrm{m}^{\prime \prime}$ für die Zuordnung, so wie sie in $\S 2$ bei den Fallunterscheidungen getroffen wurde. Die Vorverschiebungen werden dem kontinuumstheoretisch berechneten Modell entnommen. Ihre Differenzen erreichen für Punkte, die durch die Gleitebene getrennt werden, in größerem Abstand vom Kern gerade $\mathfrak{v}-\mathfrak{v}=d e_{2}$. Teilen wir, dem Beispiel in (II), $\S 8$, folgend, unseren Kristall in vier Bereiche, so können wir für die Untersuchungen jetzt unmittelbar (30) und (31) benutzen. Mit diesen beiden Gleichungen lassen sich sehr rasch die erforderlichen Aussagen über die Verzerrungsenergie der Schraubenversetzung machen. Als ersten haben wir den

Außenbereich ohne Schnitt.

Hier gilt für einen Punkt i die Formel (30). Bedenken wir, daß nach (II) (35) die Zusatzverschiebungen $\vec{\zeta}_{\mathrm{m}}-\vec{\zeta}_{\mathrm{i}}$ verschwinden, außerdem in diesem Bereich auch die $\mathfrak{k}_{\mathrm{im}}^{\mathrm{h}}$ und $P_{\mathrm{im}}^{\mathrm{h}}$ vernachlässigbar sind, so verbleibt nur das Glied

$$
\Delta U_{\mathrm{i}}=\frac{1}{2} \sum_{\mathrm{m}}\left[\mathfrak{v}_{\mathrm{i}}-\mathfrak{v}_{\mathrm{m}}\right] \cdot \boldsymbol{A}_{\mathrm{im}} \cdot\left[\mathfrak{v}_{\mathrm{m}}-\mathfrak{v}_{\mathrm{i}}\right] .
$$

Wir machen dabei von der Annahme $\mathfrak{v}_{\mathfrak{m}}-\mathfrak{v}_{\mathfrak{i}}=0$ in diesem Bereich keinen Gebrauch. Denn die Aufsummation aller Glieder (32) über $\mathrm{i}$ gibt trotz verschwindend kleiner Vorverschiebungsdifferenzen $\mathfrak{v} m-\mathfrak{v}_{\mathfrak{i}}$ im Außenbereich sehr wohl einen endlichen Energiebetrag. Genau auf die gleiche Weise erfassen wir den

Außenbereich mit Schnitt.

Hier ist Formel (31) zuständig. Aus (II) (43) ergibt sich wieder $\overrightarrow{\zeta_{\mathrm{m}}}-\overrightarrow{\zeta_{\mathrm{i}}}=0$. So verbleibt diesmal nur

$$
\begin{aligned}
\Delta U_{\mathrm{i}}=\sum_{\mathrm{m}^{\prime}} \frac{1}{2}\left[\mathfrak{v}_{\mathrm{i}}-\mathfrak{v}_{\mathrm{m}}\right] \cdot \boldsymbol{A}_{\mathrm{im}} \cdot\left[\mathfrak{v}_{\mathrm{m}}-\mathfrak{v}_{\mathrm{i}}\right] \\
\quad+\sum_{\mathrm{m}^{\prime \prime}} \frac{1}{2}\left[\mathfrak{w}_{\mathrm{i}}-\mathfrak{w}_{\mathrm{m}}\right] \cdot \boldsymbol{A}_{\mathrm{im}} \cdot\left[\mathfrak{w}_{\mathrm{m}}-\mathfrak{w}_{\mathrm{i}}\right],
\end{aligned}
$$

wo zwar die $\mathfrak{v}_{\mathfrak{m}}-\mathfrak{v}_{\mathfrak{i}}$ und $\mathfrak{w}_{\mathfrak{m}}-\mathfrak{w}_{\mathfrak{i}}$ für die Wechselwirkungsumgebung des herausgegriffenen Punktes wieder vernachlässigbar klein sind, bei einer Gesamtsummation aber berücksichtigt werden müssen. Wir erkennen, daß der Außenbereich mit Schnitt die Energieberechnung nicht andersartig beeinflußt.

Wir kommen nun zu den Innenbereichen. Hier lassen sich noch weitere Unterscheidungen treffen. Für den

\section{Innenbereich ohne Schnitt}

ist wieder Gl. (30) zuständig. Doch gibt es hier sicherlich Teilbereiche, für die zwar $\vec{\zeta}_{\mathrm{m}}-\vec{\zeta}_{\mathrm{i}}$ von Null verschieden, dagegen die höheren Glieder $\mathfrak{f}_{\mathrm{im}}^{\mathrm{h}}$ und $P_{\mathrm{im}}^{\mathrm{h}}$ noch immer vernachlässigbar sind. Dann ist neben dem quadratischen Glied der Form (32) auch noch das bilineare Glied zu berücksichtigen. Wir finden

$$
\begin{aligned}
\Delta U_{\mathrm{i}}=\sum_{\mathrm{m}}\left\{\frac{1}{2}\left[\mathfrak{v}_{\mathrm{i}}-\mathfrak{v}_{\mathrm{m}}\right]\right. & \cdot \boldsymbol{A}_{\mathrm{im}} \cdot\left[\mathfrak{v}_{\mathrm{m}}-\mathfrak{v}_{\mathrm{i}}\right] \\
& \left.+\frac{1}{2}\left[\mathfrak{v}_{\mathrm{i}}-\mathfrak{v}_{\mathrm{m}}\right] \cdot \boldsymbol{A}_{\mathrm{im}} \cdot\left[\vec{\zeta}_{\mathrm{m}}-\vec{\zeta}_{\mathrm{i}}\right]\right\} .
\end{aligned}
$$

Sind die höheren Glieder nicht vernachlässigbar, so sind wir gezwungen, auf die ursprünglichen Potentiale zu. rückzugreifen, d. h. auf

$$
\Delta U_{\mathrm{i}}=\sum_{\mathrm{m}}\left\{P_{\mathrm{im}}\left(\mathfrak{v}_{\mathrm{m}}-\mathfrak{v}_{\mathrm{i}}+\vec{\zeta}_{\mathrm{m}}-\overrightarrow{\zeta_{\mathrm{i}}}-P_{\mathrm{im}}^{0}\right\},\right.
$$

was sich auch ganz einfach dadurch ergibt, daß man in (30) die höheren Glieder der Kräfte und Potentiale durch die ursprünglichen Kräfte und Potentiale ausdrückt und berücksichtigt, daß sich in der Gesamtsumme für die Energie die Glieder

$$
\frac{1}{2} \mathfrak{f}_{i m}\left(\mathfrak{v}_{m}-\mathfrak{v}_{i}+\vec{\zeta}_{m}-\vec{\zeta}_{i}\right) \cdot\left[\zeta_{i}-\zeta_{m}\right]
$$

aus Stabilitätsgründen wegheben müssen. Wir gehen darauf nicht näher ein, sondern wenden uns dem

Innenbereich mit Schnitt

zu. Für Formel (31) gibt es entsprechend wieder beide Möglichkeiten. Wir schreiben sie der Vollständigkeit halber hier noch an

1) $\Delta U_{\mathrm{i}}=\sum_{\mathrm{m}^{\prime}}\left\{\frac{1}{2}\left[\mathfrak{v}_{\mathrm{i}}-\mathfrak{v}_{\mathrm{m}}\right] \cdot \boldsymbol{A}_{\mathrm{im}} \cdot\left[\mathfrak{v}_{\mathrm{m}}-\mathfrak{v}_{\mathrm{i}}\right]+\frac{1}{2}\left[\mathfrak{v}_{\mathrm{i}}-\mathfrak{v}_{\mathrm{m}}\right] \cdot \boldsymbol{A}_{\mathrm{im}} \cdot\left[\vec{\zeta}_{\mathrm{m}}-\vec{\zeta}_{\mathrm{i}}\right]\right\}$

$$
+\sum_{\mathrm{m}^{\prime \prime}}\left\{\frac{1}{2}\left[\mathfrak{w}_{\mathrm{i}}-\mathfrak{w}_{\mathrm{m}}\right] \cdot \boldsymbol{A}_{\mathrm{im}} \cdot\left[\mathfrak{w}_{\mathrm{m}}-\mathfrak{w}_{\mathrm{i}}\right]-\frac{1}{2}\left[\mathfrak{w}_{\mathrm{i}}-\mathfrak{w}_{\mathrm{m}}\right] \cdot \boldsymbol{A}_{\mathrm{im}} \cdot\left[\vec{\zeta}_{\mathrm{m}}-\vec{\zeta}_{\mathrm{i}}\right]\right\}
$$

und, wenn die höheren Glieder nicht vernachlässigbar sind, einfach

$$
\Delta U_{\mathrm{i}}=\sum_{\mathrm{m}^{\prime}}\left\{P_{\mathrm{im}}\left(\mathfrak{v}_{\mathrm{m}}-\mathfrak{v}_{\mathrm{i}}+\vec{\zeta}_{\mathrm{m}}-\vec{\zeta}_{\mathrm{i}}\right)-P_{\mathrm{im}}^{0}\right\}+\sum_{\mathrm{m}^{\prime \prime}}\left\{P_{\mathrm{i} \mathrm{m}}\left(-\mathfrak{w}_{\mathrm{m}}+\mathfrak{w}_{\mathrm{i}}+\vec{\zeta}_{\mathrm{m}}-\vec{\zeta}_{\mathrm{i}}\right)-P_{\mathrm{i} \mathrm{m}}^{0}\right\} .
$$

Damit sind alle Bereiche der Schraubenversetzung erfaßt und für die numerische Berechnung zugänglich ge- macht. Eine Entscheidung, wo die Außenbereiche aufhören und die Innenbereiche beginnen, kann erst nach 
Ermittlung der Zusatzverschiebungen $\vec{\zeta}_{\mathrm{m}}$ aus der gitterstatischen Rechnung gefällt werden ${ }^{8}$. Die Gesamtheit der Glieder (32) und (33) sind identisch mit der Energie des Vorverschiebungsfeldes außerhalb eines Zylinders um die Schraubenachse. Sie entspricht der Verzerrungsenergie, die wir aus kontinuumstheoretischen Rechnungen erhalten. Die sämtlichen Ausdrücke (32), (33), (35) und (37) zusammen ergeben die Energie der Schraubenversetzung.

\section{$\S$ 6. Verzerrungsenergie der Stufenversetzung}

Als Ausgangspunkt zur Berechnung der Schraubenversetzung hatten wir einen idealen Kristall gewählt, den wir durch geeignet definierte Vorverschiebungen teilweise plastisch deformierten. Bei der Stufenversetzung ist dagegen der Ausgangspunkt kein idealer, sondern ein aufgeschlitzter Kristall. Alle gitterstatischen Rechnungen haben aber von der Idealmatrix auszugehen. Somit sind wir gezwungen, auf irgendeine Weise die Entwicklungsorte der Teilchenpotentiale dieses aufgeschlitzten Kristalls auf eine ideale Anordnung abzubilden. Hierzu gibt es zwei Möglichkeiten, die in (II) und (IV) ausführlich entwickelt wurden.

Für die Energieberechnung schließen wir uns dem in (IV) entwickelten Verfahren zur Konstruktion der Stu- fenversetzung an. Ausgangspunkt für die Untersuchungen sind dort die Gln. (IV) (7-9), die zuerst mit Hilfe eines Translationstheorems auf eine für die Rechnung günstige Ausgangsform gebracht werden. In einem zweiten Schritt wird die Translation vervollständigt und das ideale Gleichungssystem erreicht. Genau diese Schritte sind jetzt im Potential selbst zu vollziehen, doch können wir sie beide zusammenfassen zu einem einzigen, da es ja hier nicht mehr um die Untersuchung der Kräfte und Zwangskräfte geht, sondern allein um die Herstellung einer idealen Anordnung der Entwicklungsorte.

Die zu (IV) (7-9) analogen Potentialgleichungen lauten ${ }^{9}$

$$
\begin{aligned}
U_{(i j)} & \left.=\sum_{\substack{m, n \\
P_{(i j)(m n)}}} \overline{(}_{m n}-\mathfrak{s}_{i j}\right) \\
& \neq m=0, n<0 \\
& (i, j) \neq(i=0, j<0) .
\end{aligned}
$$

Führen wir jetzt die Translation der Entwicklungsorte von Bereich (R) durch, so verschwindet der Spalt in (38) zwischen den Bereichen (L) und (R), und wir erhalten eine ideale Anordnung aller Entwicklungsorte. Da aber die Numerierung der Auslenkungen immer noch auf das Ausgangsmodell bezogen bleibt, läßt sich, wie in (IV), eine Aufspaltung des Gesamtgleichungssystems in die Teilsysteme für die drei Bereiche (O), (L) und (R) nicht umgehen. Sie lautet:

1) $(i, j)$ in Bereich $(0)$ :

$$
\left.U_{(i j)}=\sum_{m, n \geq 0} \bar{P}_{(i j)(m n)}\left(\mathfrak{s}_{m n}-\mathfrak{s}_{i j}\right)+\sum_{m<0, n<0} \bar{P}_{(i j)(m n)}\left(\mathfrak{s}_{m n}-\mathfrak{s}_{i j}\right)+\sum_{m>0, n<0} \boldsymbol{P}_{(i j)(m-1, n)}\right)\left(\mathfrak{s}_{m n}-\mathfrak{s}_{i j}+\mathfrak{e}_{\mathbf{1}} d\right),
$$

2) $(i, j)$ in Bereich (L) :

$$
\left.U_{i j}=\sum_{m, n \geq 0} P_{(i j)(m n)}\left(\mathfrak{s}_{m n}-\mathfrak{s}_{i j}\right)+\sum_{m<0, n} \bar{P}_{(i j)(m n)}\left(\mathfrak{s}_{m n}-\mathfrak{s}_{i j}\right)+\sum_{m>0, n<0} \bar{P}_{(i j)(m-1, n}\right)\left(\mathfrak{s}_{m n}-\mathfrak{s}_{i j}+\mathfrak{e}_{\mathbf{1}} d\right),
$$

3) $(i, j)$ in Bereich $(\mathrm{R})$ :

$$
\left.U_{i j}=\sum_{m, n \geq 0} \bar{P}_{(i-1, j)(m n)}\left(\mathfrak{s}_{m n}-\mathfrak{s}_{i j}-\mathfrak{e}_{1} d\right)+\sum_{m<0, n<0} \bar{P}_{(i-1, j)(m n)}\left(\mathfrak{s}_{m n}-\mathfrak{s}_{i j}-\mathfrak{e}_{1} d\right)+\underset{m>0, n<0}{\sum_{(i-1, j)(m-1, n)}} \overline{\mathbf{P}}_{m n n}-\mathfrak{s}_{i j}\right) .
$$

Wir ersetzen in (39) die Freiheitsgrade $\mathfrak{s}_{m n}$ durch neue Variable $\vec{\xi}_{m n}$ mit Hilfe der Definitionen

$$
\vec{\xi}_{m n}=\mathfrak{s}_{m n} \quad \text { in }(\mathrm{O}) \text { und (L), } \quad \vec{\xi}_{m n}=\mathfrak{s}_{m n}+\mathfrak{e}_{1} d \quad \text { in }(\mathrm{R}) .
$$

Dann verbleibt in jedem Glied der drei Systeme (39) nur noch das Argument $\left(\vec{\xi}_{m n}-\vec{\xi}_{i j}\right)$. Die Entwicklung nach diesen Auslenkungsdifferenzen ist für unsere Betrachtungen wesentlich. Wir geben sie daher vollständig an. 1) $(i, j)$ in $(0)$ :

$$
\begin{aligned}
\Delta U_{(i j)=\sum_{m, n \geq 0}\left\{\frac{1}{2}\left[\vec{\xi}_{i j}-\vec{\xi}_{m n}\right] \cdot \boldsymbol{A}\right.}(i j)(m n) & \cdot\left[\vec{\xi}_{m n}-\vec{\xi}_{i j}\right]+P_{(i j)(m n)}^{\mathrm{h}}\left(\vec{\xi}_{m n}-\vec{\xi}_{i j)}\right\} \\
& +\sum_{m}\left\{\frac{1}{2}\left[\vec{\xi}_{i j}-\vec{\xi}_{m n}\right] \cdot \overrightarrow{\boldsymbol{A}}_{(i j)(m n)} \cdot\left[\vec{\xi}_{m n}-\vec{\xi}_{i j}\right]+\vec{P}_{(i j)(m n)}^{\mathrm{h}}\left(\vec{\xi}_{m n}-\vec{\xi}_{i j)}\right\}\right. \\
& +\sum_{m>0, n}\left\{\frac{1}{2}\left[\vec{\xi}_{i j}-\vec{\xi}_{m n}\right] \cdot \overrightarrow{\boldsymbol{A}}_{(i j)(m-1, n)} \cdot\left[\vec{\xi}_{m n}-\vec{\xi}_{i j}\right]+\overrightarrow{\boldsymbol{P}}_{(i j)(m-1, n)}^{\mathrm{h}}\left(\vec{\xi}_{m n}-\vec{\xi}_{i j}\right\} .\right.
\end{aligned}
$$

2) $(i, j)$ in (L). Die Formel lautet genau wie $(41 \mathrm{a})$,

8 Für die der Schraubenachse unmittelbar benachbarten Ionenreihen können Zweifel bestehen, zu welchem Bereich man sie zählen soll. Da hier die Vorverschiebungen und deren Ergänzungsvektoren praktisch gleichgroß sind und man sowieso die Glieder höherer Ordnung dort vollständig benutzen muß, ist es gleichgültig, ob man sie dem Innenbereich mit Schnitt oder ohne Schnitt zuordnen will.

9 Bezüglich der Bereichseinteilung siehe (IV). Die Bezeichnungsweise wird von dort übernommen. 
3) $(i, j)$ in $(\mathrm{R})$ :

$$
\begin{aligned}
& \Delta U_{(i j)}=\sum_{m, n \geq 0}\left\{\frac{1}{2}\left[\vec{\xi}_{i j}-\vec{\xi}_{m n}\right] \cdot \overrightarrow{\boldsymbol{A}}_{(i-1, j)(m n)} \cdot\left[\vec{\xi}_{m n}-\vec{\xi}_{i j}\right]+\vec{P}_{(i-1, j)(m n)}^{\mathrm{h}}\left(\vec{\xi}_{m n}-\vec{\xi}_{i j}\right)\right\} \\
&+\sum_{m<0, n<0}\left\{\frac{1}{2}\left[\vec{\xi}_{i j}-\vec{\xi}_{m n}\right] \cdot \overrightarrow{\boldsymbol{A}}_{(i-1, j)(m n)} \cdot\left[\vec{\xi}_{m n}-\vec{\xi}_{i j}\right]+P_{(i-1, j)(m n)}^{\mathrm{h}}\left(\vec{\xi}_{m n}-\vec{\xi}_{i j}\right)\right\} \quad(41 \mathrm{c}) \\
&+\sum_{m>0 . n<0}\left\{\frac{1}{2}\left[\vec{\xi}_{m n}-\vec{\xi}_{i j}\right] \cdot \overrightarrow{\boldsymbol{A}}_{(i-1, j)(m-1, n)} \cdot\left[\vec{\xi}_{m n}-\vec{\xi}_{i j}\right]+P_{(i-1, j)(m-1, n)}^{\mathrm{h}}\left(\vec{\xi}_{m n}-\vec{\xi}_{i j}\right)\right\} .
\end{aligned}
$$

Die linearen Glieder verschwinden, da ja eine ideale Anordnung vorliegt ${ }^{10}$.

Neben diese Entwicklungen (41) stellen wir die Kraftgleichungen (IV) $(17-19)$. Allerdings haben wir die letzteren vor dem Vergleich noch auf die Variablen $\vec{\xi}_{m n}$ zu transformieren ${ }^{11}$. Sie lauten dann:

1) Für $(i, j)$ im Bereich (0)

$$
\begin{aligned}
& \sum_{m, n \geq 0} \boldsymbol{A}_{(i j)(m n)} \cdot\left[\vec{\xi}_{m n}-\vec{\xi}_{i j}\right]+\sum_{m<0, n<0} \overrightarrow{\boldsymbol{A}}_{(i j)(m n)} \cdot\left[\vec{\xi}_{m n}-\vec{\xi}_{i j}\right]+\sum_{m>0, n<0} \overrightarrow{\boldsymbol{A}}_{(i j)(m n)} \cdot\left[\vec{\xi}_{m n}-\vec{\xi}_{i j}\right] \\
& \quad=\sum_{m>0, n<0}\left\{\mathfrak{R}_{(i j)(m-1, n)}-\overline{\mathfrak{K}}_{(i j)(m n)}+\left[\overrightarrow{\boldsymbol{A}}_{(i j)(m-1, n)}-\overline{\boldsymbol{A}}_{(i j)(m n)}\right] \cdot\left[\vec{\xi}_{m n}-\vec{\xi}_{i j}-\mathfrak{e}_{1} d\right]\right\} \\
& \quad+\sum_{m>0, n<0} \boldsymbol{A}_{(i j)(m-1, n)} \cdot \mathfrak{e}_{1} d-\sum_{m, n \geq 0} \mathfrak{\mathfrak { f }}_{(i j)(m n)}^{\mathrm{h}}\left(\vec{\xi}_{m n}-\vec{\xi}_{i j}\right)-\sum_{m<0, n<0} \mathfrak{\mathfrak { f }}_{(i j)(m n)}^{\mathrm{h}}\left(\vec{\xi}_{m n}-\vec{\xi}_{i j}\right)-\sum_{m>0, n<0} \mathfrak{f}_{(i j)(m n)}^{\mathrm{h}}\left(\vec{\xi}_{m n}-\vec{\xi}_{i j}-\mathfrak{e}_{1} d\right)=\mathfrak{\xi}_{i j}^{(0)},
\end{aligned}
$$

2) Für $(i, j)$ in Bereich (L) :

$$
\begin{gathered}
\sum_{m, n \geq 0} \boldsymbol{A}_{(i j)(m n)} \cdot\left[\vec{\xi}_{m n}-\vec{\xi}_{i j}\right]+\sum_{m<0, n<0} \overline{\boldsymbol{A}}_{(i j)(m n)} \cdot\left[\vec{\xi}_{m n}-\vec{\xi}_{i j}\right]+\sum_{m>0, n<0} \boldsymbol{A}_{(i j)(m-1, n)} \cdot\left[\vec{\xi}_{m n}-\vec{\xi}_{i j}\right] \\
=-\sum_{m, n \geq 0} \mathfrak{\mathfrak { f }}_{(i j)(m n)}^{\mathrm{h}}\left(\vec{\xi}_{m n}-\vec{\xi}_{i j}\right)-\sum_{m<0,} \mathfrak{f}_{(i j)(m n)}^{\mathrm{h}}\left(\vec{\xi}_{m n}-\vec{\xi}_{i j}\right)-\sum_{m>0, n<0} \mathfrak{f}_{(i j)(m-1, n)}^{\mathrm{h}}\left(\vec{\xi}_{m n}-\vec{\xi}_{i j}-\mathfrak{e}_{1} d\right)=\overrightarrow{\mathfrak{F}}_{i j}^{(\mathrm{L})},
\end{gathered}
$$

3) Für $(i, j)$ in Bereich (R) :

$$
\begin{aligned}
\sum_{m, n \geq 0} \overrightarrow{\boldsymbol{A}}_{(i-1, j)(m n)} \cdot\left[\vec{\xi}_{m n}-\vec{\xi}_{i j}\right]+\sum_{m<0, n<0} \boldsymbol{A}_{(i-1, j)(m n)} \cdot\left[\vec{\xi}_{m n}-\vec{\xi}_{i j}\right]+\sum_{m>0, n<0} \overrightarrow{\boldsymbol{A}}_{(i-1, j)(m-1, n)} \cdot\left[\vec{\xi}_{m n}-\vec{\xi}_{i j}\right] \\
\quad=\sum_{m, n \geq 0}\left\{\boldsymbol{A}_{(i-1, j)(m n)}-\overrightarrow{\boldsymbol{A}}_{(i j)(m n)}\right\} \cdot\left[\vec{\xi}_{m n}-\vec{\xi}_{i j}\right]-\sum_{m, n \geq 0} \overrightarrow{\boldsymbol{A}}_{(i-1, j)(m n)} \cdot \mathfrak{e}_{\mathbf{1}} d \\
\quad-\sum_{m, n \geq 0} \mathfrak{f}_{(i j)(m n)}^{\mathrm{h}}\left(\vec{\xi}_{m n}-\vec{\xi}_{i j}+\mathfrak{e}_{\mathbf{1}} d\right)-\sum_{m<0, n<0} \mathfrak{f}_{(i-1, j)(m n)}^{\mathrm{h}}\left(\vec{\xi}_{m n}-\vec{\xi}_{i j}+\mathfrak{e}_{1} d\right)-\sum_{m>0, n<0} \mathfrak{f}_{(i j)(m n)}^{\mathrm{h}}\left(\vec{\xi}_{m n}-\vec{\xi}_{i j}\right)=\overrightarrow{\mathfrak{F}}_{i j}^{(\mathrm{R})} .
\end{aligned}
$$

Beim Vergleich der linken Seiten von (42) mit (41) erkennen wir sofort die Möglichkeit der Reduktion unserer Energieformel durch Multiplikation der Kräfte (42) mit $\vec{\xi}_{i j}$ und anschließender Umordnung. Wir erhalten demnach für die Verzerrungsenergie, wenn wir die Energie des Idealkristalls $\frac{1}{2} \sum_{i j} P_{i j}{ }^{0}$ mit auf die linke Seite nehmen

$$
\Delta U=\frac{1}{2} \sum_{i j}\left\{U_{i j}-P_{i j}{ }^{0}\right\}=\sum_{i, j \geq 0} \frac{1}{2} \vec{\xi}_{i j} \cdot \mathfrak{k}_{i j}^{(0)}+\sum_{i<0, j<0} \frac{1}{\frac{1}{2}} \vec{\xi}_{i j} \cdot \mathfrak{k}_{i j}^{(\mathrm{L})}+\sum_{i>0, j<0} \frac{1}{2} \vec{\xi}_{i j} \cdot \mathfrak{k}_{i j}^{(\mathrm{R})}+\frac{1}{2} \overline{P^{\mathrm{h}}} .
$$

(43) ist die Ausgangsform der Energieberechnung für die Stufenversetzung, wenn wir uns dem Erzeugungsverfahren in (IV) anschließen.

In (43) ist jetzt nur noch das Glied $\frac{1}{2} P^{\mathrm{h}}$ zu bestimmen. Es enthält die Gesamtheit der Glieder höherer Ordnung, die wir erhalten, wenn wir alle $\bar{P}_{(i j)(m n)}\left(\vec{\xi}_{m n}-\vec{\xi}_{i j}\right)$ in $(41)$ über $(i, j)$ summieren. Man darf allerdings nicht annehmen, daß diese, genauso wie die höheren Kraftglieder $\mathfrak{f}_{(i j)(m n)}^{h}$, außerhalb des Kerns durchweg vernachlässigbar klein seien. Dies trifft auf keinen Fall zu entlang der Gleitebene, die in unserem Fall annähernd mit der positiven $x_{1}$-Achse zusammenfällt. Dort nämlich erreichen die Auslenkungsdifferenzen die Größenordnung

$$
\vec{\xi}_{m n}-\vec{\xi}_{i j} \approx \pm e_{1} d
$$

10 Man darf sich durch die Art der Abzählung in (41) nicht stören lassen. Dort wurde nämlich die Benennung nach den Ausgangslagen im aufgeschlitzten Kristall beibehalten. Man könnte durch Umbenennung und Umindizierung na- türlich sofort die geschlossene Darstellung in einer Gleichung erreichen.

11 Die Umbenennung (IV) (16) machen wir in (IV) (17) bis (19) rückgängig. 
sofern $(m, n)$ und $(i, j)$ durch den Schnitt zwischen Bereich (O) und (R) getrennt sind. So verbleiben für $\bar{P}^{\mathrm{h}}$ mindestens eine Anzahl Glieder aus dem folgenden Ausdruck

$$
\bar{P}^{\mathrm{h}}=\sum_{i, j \geq 0} \sum_{m>0,} P_{n<0}^{\mathrm{h}}\left(_{(i j)(m-1, n)}\left(\vec{\xi}_{m n}-\vec{\xi}_{i j}\right)+\sum_{i>0, j<0} \sum_{m, n \geq 0} P_{(i-1, j)(m, n)}^{\mathrm{h}}\left(\vec{\xi}_{m n}-\vec{\xi}_{i j}\right)+\sum_{k, l} P_{(k l)}^{\mathrm{h}} .\right.
$$

$\bar{P}_{(k l)}^{\mathrm{h}}$ soll dabei die höheren Glieder andeuten, die wir im Kern außerdem noch berücksichtigen müssen. Mit (44) geben die beiden Summen in (45) entlang des Schnitts auch in beliebiger Entfernung vom Kern sicherlich noch Beiträge zur Gesamtenergie. Dagegen lassen sich alle weiteren Glieder höherer Ordnung außerhalb des Kerns vernachlässigen.

Wir sind damit auch für die Stufenversetzung am Ziel unserer Betrachtungen. Für eine spätere Diskussion der Energiebeiträge am speziell berechneten Modell der Stufenversetzung im Ionenkristall ist es jedoch notwendig, eine Zusammenfassung der Kräfte (42) und der höheren Potentialglieder (45) in der Energiegleichung (43) zu betrachten. Dazu drücken wir $P^{\mathrm{h}}$ durch die ursprünglichen Potentiale aus und finden

$$
\begin{aligned}
\bar{P}^{\mathrm{h}}=\sum_{i, j \geq 0} & \sum_{m>0, n<0}\left\{\overline{\boldsymbol{P}}_{(i j)(m-1, n)}\left(\vec{\xi}_{m n}-\vec{\xi}_{i j}\right)-\vec{P}_{(i j)(m-1, n)}^{(0)}-\left[\vec{\xi}_{i j}-\vec{\xi}_{m n}\right] \cdot \overrightarrow{\mathcal{R}}_{(i j)(m-1, n)}\right. \\
& \left.\quad-\frac{1}{2}\left[\vec{\xi}_{i j}-\vec{\xi}_{m n}\right] \cdot \overrightarrow{\boldsymbol{A}}_{(i j)(m-1, n)} \cdot\left[\vec{\xi}_{m n}-\vec{\xi}_{i j}\right]\right\}+\sum_{i>0, j<0} \sum_{m, n \geq 0}\left\{\vec{P}_{(i-1, j)(m n)} \vec{\xi}_{m n}-\vec{\xi}_{i j}\right)-\overline{\boldsymbol{P}}_{(i-1, j)(m n)}^{(0)} \\
& \left.-\left[\vec{\xi}_{i j}-\vec{\xi}_{m n}\right] \cdot \overrightarrow{\mathcal{K}}_{(i-1, j)(m n)}-\frac{1}{2}\left[\vec{\xi}_{i j}-\vec{\xi}_{m n}\right] \cdot \overrightarrow{\boldsymbol{A}}_{(i-1, j)(m n)}\left[\vec{\xi}_{m n}-\vec{\xi}_{i j}\right]\right\} .
\end{aligned}
$$

Dies in (43) eingetragen, gibt bei Berücksichtigung von (42) ${ }^{12}$ und der Beziehungen

$$
\begin{array}{rr}
\overline{\boldsymbol{A}}_{(m-1, n)(i j)}=\overline{\boldsymbol{A}}_{(i j)(m-1, n)} ; & \overline{\boldsymbol{P}}_{(m-1, n)(i j)}\left(\vec{\xi}_{m n}-\vec{\xi}_{i j}\right)=\overline{\boldsymbol{P}}_{(i j)(m-1, n)}\left(\vec{\xi}_{m n}-\vec{\xi}_{i j)} ;\right. \\
P_{(m-1, n)(i j)}^{(0)}=\overline{\boldsymbol{P}}_{(i j)(m-1, n)}^{(0)} ; & \overline{\boldsymbol{R}}_{(m-1, n)(i j)}=-\overline{\mathfrak{R}}_{(i j)(m-1, n)}
\end{array}
$$

für die Energie den Ausdruck

$$
\begin{aligned}
\Delta U & =\frac{1}{2} \sum_{i, j \geq 0} \sum_{m>0, n<0}\left\{\vec{\xi}_{i j}\left[\Re_{(i j)(m-1, n)}-\mathfrak{R}_{(i j)(m n)}\right]+\left[\vec{\xi}_{i j}-\vec{\xi}_{m n}\right]\left[\overrightarrow{\boldsymbol{A}}_{(i j)(m-1, n)}-\overrightarrow{\boldsymbol{A}}_{(i j)(m n)}\right]\left[\vec{\xi}_{m n}-\vec{\xi}_{i j}\right]\right. \\
& -\vec{\xi}_{i j} \cdot\left[\overline{\boldsymbol{A}}_{(i j)(m-1, n)}-\overline{\boldsymbol{A}}_{(i j)(m n)}\right] \cdot \mathfrak{e}_{1} d-2\left[\vec{\xi}_{i j}-\vec{\xi}_{m n}\right] \cdot \overrightarrow{\mathcal{R}}_{(i j)(m-1, n)}+\left[\vec{\xi}_{i j}-\vec{\xi}_{m n}\right] \cdot \overline{\boldsymbol{A}}_{(i j)(m-1, n)} \cdot\left[d \mathrm{e}_{1}-\left(\vec{\xi}_{m n}-\vec{\xi}_{i j}\right)\right] \\
& \left.+2 \bar{P}_{(i j)(m-1, n)} \vec{\xi}_{m n}-\vec{\xi}_{i j)}-2 \vec{P}_{(i j)(m-1, n)}^{(0)}\right\}+\frac{1}{2} \sum_{k, l}\left\{-\overrightarrow{\boldsymbol{\xi}}_{(k l)}^{\mathrm{h}} \cdot \vec{\xi}_{k l}+\overline{\boldsymbol{P}}_{(k l)}^{\mathrm{h}}\right\} .
\end{aligned}
$$

Mit (49) haben wir die ausgeschriebene Formel für die Energieberechnung des Stufenversetzungsmodells aus (IV). In dieser Form ist sie allerdings noch recht schwerfällig. Transformiert man nachträglich auf die ursprünglichen Freiheitsgrade $\mathfrak{s}_{m n}$ zurück, dann erhält man eine erhebliche Vereinfachung. Wir unterdrücken jedoch diese Transformation hier, da wir bei der numerischen Berechnung der Versetzungsenergie später diese Formel ohnehin noch einer näheren Betrachtung unterziehen müssen.

Úber die Anteile der einzelnen Glieder in (49) für den Kern der Versetzung können wir von diesem allgemeinen Standpunkt aus noch nicht sehr viel aussagen. Nach (44) ist auch das Verhalten in größerer Entfernung vom Kern von Interesse. Eine genauere Untersuchung zeigt, daß sich in der Zusammenfassung (48) die Energiebeiträge entlang des Schnitts, sobald der Kristall dort wieder eine annähernd ideale Struktur erreicht hat, gegenseitig wegheben. $\mathrm{Da}$ wir jedoch keine endliche Gesamtenergie, sondern eine logarithmisch di- vergierende erhalten, beruht auf der logarithmischen Divergenz der Auslenkungen am Rande des Kristalls. Die Diskussion dieser Einzelfragen verschieben wir, bis die numerischen Ergebnisse für die Stufenversetzung vorliegen.

Herrn Prof. E. Fues danke ich herzlich für die Unterstützung dieser Arbeit und seine kritischen Bemerkungen. Besonders gilt mein Dank Herrn Dr. H. StumpF für seine Anregungen und Diskussionen zur Klärung dieser gitterstatischen Probleme. Der De u t s chen Forschungsgemeinschaft sei für die Förderung der Untersuchungen gedankt, von denen diese Arbeit ein Teil ist.

12 Wie in (IV) besonders herausgestellt wurde, war das Ausgangsmodell so gewählt worden, daß wir die höheren Kraftglieder in (42) bis auf ganz wenige vernachlässigen konnten. Wir schreiben daher in (47) nur noch symbolisch $\mathfrak{f}_{(k l)}^{\mathrm{h}}$ für die berücksichtigten höheren Glieder im Kern selbst. 